\title{
Generation and characterization of a novel transgenic mouse harboring conditional nuclear factor-kappa B/RelA knockout alleles
}

Talha ljaz ${ }^{1}$, Maki Wakamiya ${ }^{2}$, Hong Sun ${ }^{3}$, Adrian Recinos $\|^{3}$, Ronald G. Tilton ${ }^{2,3,4}$ and Allan R. Brasier ${ }^{1,2,3,4^{*}}$ (1)

\begin{abstract}
Background: Nuclear Factor-Kappa B (NF-kB) is a family of transcription factors that are important in embryonic development, inflammation, epithelial-to-mesenchymal transition and cancer. The $65 \mathrm{kDa}$ RelA subunit is the major transcriptional activator of the NF-kB pathways. Whole-body deficiency of RelA leads to massive apoptosis of liver hepatocytes and death in utero. To study the role of RelA in physiology and in disease states in a manner that circumvents this embryonic lethal phenotype, we have generated a mouse with RelA conditional knockout (CKO) alleles containing loxP sites that are deleted by activated Cre recombinase.

Results: We demonstrate that RelA ${ }^{\mathrm{CKO} / \mathrm{CKO}}$ mice are fertile, do not display any developmental defects and can be crossed with Cre-expressing mice to delete RelA in a temporal, tissue-specific manner. Our mating of RelA ${ }^{\text {CKO/CKO }}$ mice with Zp3-Cre transgenic led to embryonic lethality of RelA-deficient embryos. In contrast, mating of RelA ${ }^{\mathrm{CKO} / \mathrm{CKO}}$ mice with Col1a2-CreER mice allowed for the generation of double transgenics which could be stimulated with tamoxifen to induce fibroblast-specific RelA deletion in adulthood.

Conclusions: Based on our collective data, we conclude that this novel RelA ${ }^{\mathrm{CKO} / \mathrm{CKO}}$ mouse allows for efficient deletion of RelA in a tissue-specific manner. This RelA ${ }^{\mathrm{CKO} / \mathrm{CKO}}$ mouse will be an invaluable tool for deciphering the mechanistic roles of RelA in various cells and tissues during development and in disease.
\end{abstract}

Keywords: NF-kB, RelA, p65, Flox, Tamoxifen, Cre, Col1a2

\section{Background}

$\mathrm{NF}-\mathrm{kB}$ is an inducible transcription factor complex involved in the regulation of genes necessary for cell survival, differentiation, immunity and inflammation [1]. RelA, C-Rel and RelB are transcriptional activating subunits that contain a N-terminal Rel homology domain (RHD) and a C-terminal transcription activation domain (TAD) whereas NF-kB1 (p50/p105) and NF-kB2 (p52/ p100) are DNA-binding proteins that contain C-terminal autoinhibitory ankyrin repeat domains [2]. In most cells, NF-kB is high molecular weight heterodimeric

\footnotetext{
* Correspondence: arbrasie@utmb.edu

${ }^{1}$ Departments of Biochemistry and Molecular Biology, University of Texas Medical Branch, Galveston, TX, USA

${ }^{2}$ Institute for Translational Sciences, University of Texas Medical Branch, Galveston, TX, USA

Full list of author information is available at the end of the article
}

complexes containing a DNA-binding- and a transcription activator subunit retained in the cytoplasm by Inhibitor of kappa B (IkB) proteins. Receptor activation by numerous ligands induces phosphorylation of $\mathrm{IkB} \alpha$, targeting it for degradation and allowing nuclear translocation of RelA.NF-kB1 dimers, the most abundant and most potent transcription factor pair required for activation of inflammatory and anti-apoptotic gene expression programs [3].

Beg and colleagues were the first to describe the lethality of whole-body RelA deficiency in mice [4]. Mating of RelA heterozygous mice failed to generate any RelA-deficient $\left(\operatorname{RelA}^{-/}\right)$animals and an analysis of embryos indicated that RelA ${ }^{-1-}$ embryos undergo massive liver degeneration with hepatocyte apoptosis and consequently die in utero. These findings were independently 
corroborated using a different gene-targeting vector [5]. This study demonstrated that when RelA $^{-1-}$ embryonic livers are transplanted into SCID mice, $\mathrm{T}$ and $\mathrm{B}$ cells populate spleen and lymph nodes to similar extent as wild-type (WT) cells, suggesting that the lethal effect of RelA $^{-/}$in utero is due to impairment of hepatocyte development and not due to deranged hematopoiesis. In a subsequent finding, the lethal phenotype of RelA ${ }^{-1-}$ was rescued by absence of TNFa [6]. RelA activation downstream of the TNF-receptor is necessary for the production of anti-apoptotic molecules, including TRAF-1, TRAF-2, c-IAP1 AND c-IAP2 that protect cells from local, endogenously produced TNF $\alpha$-induced apoptosis $[7,8]$.

To overcome embryonic lethality produced by wholebody RelA deletion and to investigate the role of RelA in disease-specific states, investigators have generated transgenic mice inserting loxP sites in the RelA gene (RelA-floxed). DNA recombination mediated by Cre recombinase in RelA-floxed animals leads to truncation or deletion of the RelA protein. Algul and colleagues created a RelA-floxed mouse containing loxP sites in RelA introns 6 and 10 [9]. Induction of Cre led to deletion of exons 7-10, encoding the RHD, producing a truncated RelA that failed to translocate to the nucleus. This strategy results in expression of a truncated RelA C-terminal TAD that potentially confounds the study. Recently, another group created RelA-floxed mice with loxP sites flanking the promoter region and exon 1 that resulted in the deletion of RelA in B-cells when RelA-floxed animals were crossed with CD19-Cre trasngenics [10]. The strategy to excise only exon 1 of the RelA gene has the potential to allow the expression of alternatively spliced variants. In the case of RelA, there is putative internal ribosome entry site (IRES) in exons 4-5 which allows for 5 'cap-indepedant translation [11]. Additionally, there is evidence of alternatively spliced variants of RelA in different cell types. For example, p65 $\Delta$ variant lacks aa 222-231 and is abundantly expressed in pre- $\mathrm{B}$ and erythroid colony forming cells [12]. During screening of human adult osteoblastic cDNA library a RelA variant, p $65 \Delta 2$, has been found that lacks amino acids (aa) 1325 and 506 [13]. The N-terminus aa 13-25 are part of the RHD, corresponding to exon 2 and 3, and may be needed for binding to DNA. Although there has not been any exhaustive investigation into alternative RelA splicing variants, the presence of IRES in exons 4-5 and existence of RelA NH2-terminal splice variants suggests that some RelA transcripts may be produced in specialized cells under pathophysiological conditions that lack exon 1 but still can undergo translation. These splice variants would not be targeted in the flox mouse created by Heise and colleagues. Furthermore, cells that undergo recombination in the transgenic by Hesie et al. begin expressing eGFP. This strategy makes it easier to track recombined cells but the eGFP signal could be confounding in leukocytes, such as monocytes/macrophages, that have significant intracellular granules and are autofluorescent in the same emission range as GFP [14]. We decided to create a RelA-floxed mouse for investigating NF-kB signaling in vivo because, at the time, no such experimental tool was commercially available. Our group has developed a RelA-floxed transgenic mouse that allows for complete deletion of RelA and which will be made available for use by the scientific community.

The mouse RelA gene, comprised of 11 exons, is located within a $10.65-\mathrm{kb}$ region on Chromosome 19 . Our strategy was to introduce two loxP sites into intron 4 and intron 8 of the RelA gene via gene targeting [RelA conditional knockout (RelA ${ }^{C K O}$ ) allele, Fig. 1]. In the presence of Cre recombinase, a $2.7-\mathrm{kb}$ sequence containing exons $5-8$ would be excised from RelA ${ }^{\mathrm{CKO} / \mathrm{CKO}}$ generating the $\operatorname{RelA}^{-1-}$. The deletion of exons 5-8, which encode the C-terminus of its RHD (aa 113-292), results in a downstream frame-shift that will further lead to degradation of RelA transcripts via nonsense-mediated mRNA decay.

\section{Results and Discussion}

The gene-targeting vector containing an frt-flanked neomycin positive-selection cassette, and a thymidine kinase negative-selection cassette (see Methods and Fig. 1) was electroporated into 129B6F1 hybrid mouse embryonic stem (ES) cells, G4 [15]. One hundred-forty ES cell colonies survived 9-days of double-drug selection. Colonies were selected and propagated and DNA isolated from the cells was screened for homologous recombinants by Southern blotting. We found that twelve clones underwent homologous recombination at the RelA locus, and of those, three clones incorporated a "stand-alone" loxP sequence in intron 8 (Fig. 1a). After further expansion and repeated Southern blot analysis (Fig. 1b), all three clones were injected into $\mathrm{C} 57 \mathrm{BL} / 6 \mathrm{~J}$ blastocysts. We then bred the resulting male chimeras with FLPeR mice on a C57BL/6] background [B6.129S4-Gt(RO-

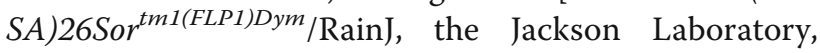
Stock Number: 009086] to obtain offspring doubleheterozygous for RelA ${ }^{C K O}$ allele and FLP1 knock-in allele. The FLPeR mice express FLP1 recombinase in a wide range of cell types including germ cells [16], and were used for FLP-mediated removal of the neomycin cassette from the mutant RelA allele (Fig. 2). Germ-line transmission of RelA mutation was achieved with two clones, 1-60 and 1-95. We then backcrossed double-heterozygous mice to $\mathrm{C} 57 \mathrm{BL} / 6 \mathrm{~J}$ mice to segregate the $F L P 1$ transgene and to establish RelA ${ }^{\mathrm{CKO}}$ lines. Mice homozygous for $\operatorname{RelA}^{\text {CKO }}$ allele (RelA ${ }^{\mathrm{CKO} / \mathrm{CKO}}$ ) were normal, fertile and expressed RelA at normal levels. 


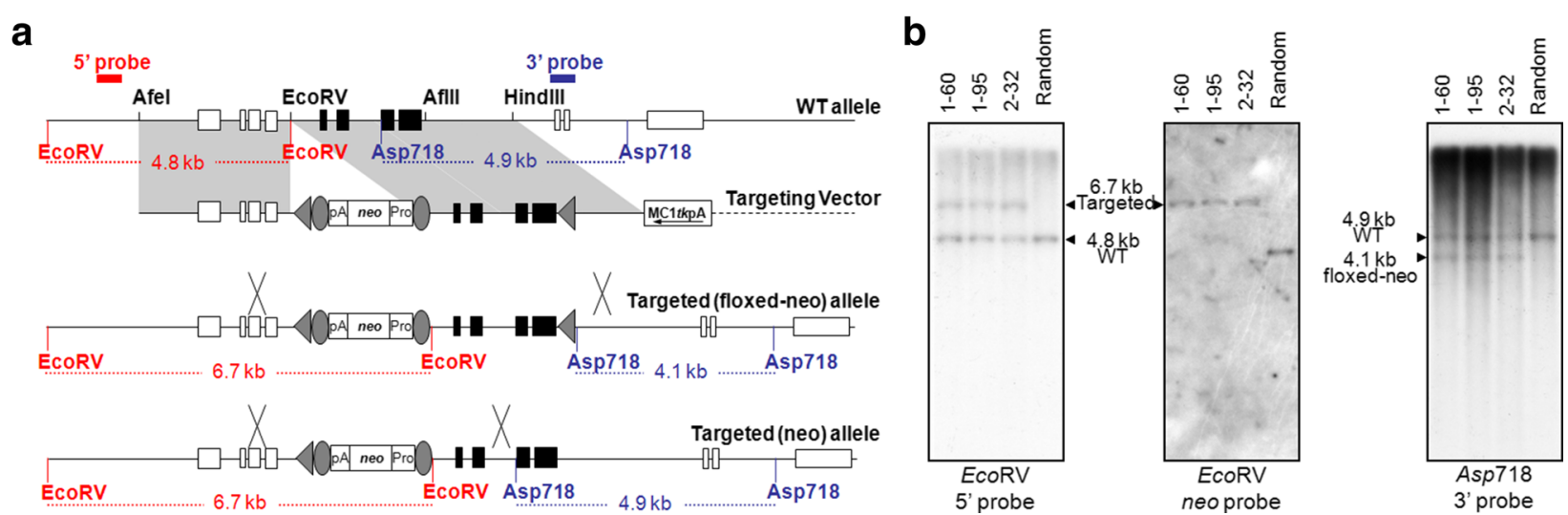

Fig. 1 Strategy for targeted mutation of the RelA gene. a The open boxes represent exons 1-4, and 9-11; the solid boxes represent exons 5-8. The solid lines represent intronic sequences; the dashed line represents plasmid vector DNA. The triangles represent loxP sequences and the ovals represent frt sequences. The gene-targeting vector possesses a total of 7.2-kb homologous sequence. The PGKneobpA cassette was inserted into an EcoRV site located $\sim 0.6-\mathrm{kb}$ upstream of exon 5. The second (stand-alone) loxP was inserted into an Aflll site located $\sim 240$-bp downstream of exon 8. The vector has a 2.9-kb 5'-homology arm, and a 3'-homology arm divided into 2.7-kb and 1.7-kb segments. Homologous recombination between WT RelA and the targeting vector may result in a RelA mutant allele that carries both PGKneobpA cassette and the stand-alone IoxP [Targeted (floxed-neo) allele], or one that carries only PGKneobpA cassette [Targeted (neo) allele]. The 5'-flanking probe hybridized to EcoRVdigested genomic DNA detects 4.8-kb WT bands, and 6.7-kb targeted allele bands; the 3'-flanking probe hybridized to Asp718-digested genomic DNA detects 4.9-kb WT bands, 4.1-kb targeted (floxed-neo) allele bands, and 4.9-kb targeted (neo) allele bands. b Southern blot analysis of DNA isolated from ES cell clones. Three clones, 1-60, 1-95, and 2-32, were found correctly targeted in the initial screening, and further expanded; DNA was isolated and subjected to Southern blot analysis. DNA isolated from a random integration clone was used as a non-recombinant control (Random). A restriction enzyme and a probe used in the analysis were indicated below each panel

To generate whole-body RelA deficient mice containing the knockout alleles [KO, Fig. 3], we first crossed a RelA ${ }^{\mathrm{CKO} /+}$ mouse (1-60 line) and a $\mathrm{Zp3} 3$-cre transgenic mouse on a C57BL/6 background [C57BL/6J- $\mathrm{Tg}(\mathrm{Zp} 3-$ cre) $93 \mathrm{Knw} / \mathrm{J}$, The Jackson Laboratory, Stock Number: 003651, [17]], and obtained a $\operatorname{RelA}{ }^{\mathrm{CKO} /+} ; \mathrm{Tg}(\mathrm{Zp} 3-$ cre) $93 \mathrm{Knw} / 0$ female mouse. In Zp3-cre transgenic mice, Cre expression is controlled by regulatory sequences from the mouse zona pellucida 3 (Zp3) gene, which is normally expressed exclusively in the growing oocytes, and Cre-mediated loxP recombination occurs in $100 \%$ of oocytes [18]. Therefore, backcrossing the RelA ${ }^{\mathrm{CKO} /+}$; $\mathrm{Tg}(\mathrm{Zp} 3-\mathrm{cre}) 93 \mathrm{Knw} / 0$ female mouse to a wild-type C57BL/6J male results in offspring: $\operatorname{RelA}^{+/-} ; \mathrm{Tg}(\mathrm{Zp} 3-$ cre) $93 \mathrm{Knw} / 0, \mathrm{RelA}^{+/+} ; \mathrm{Tg}(\mathrm{Zp} 3-\mathrm{cre}) 93 \mathrm{Knw} / 0, \mathrm{RelA}^{+/-}$and RelA $^{+/+}$. The offspring were genotyped by Southern blot (Fig. 3), and a RelA ${ }^{+/-}$mouse was backcrossed to C57BL/ 6) mice for the propagation of the $\mathrm{KO}$ line. $\mathrm{RelA}^{+/-}$male and female mating produced only $\mathrm{RelA}^{+/-}$and $\mathrm{RelA}^{+/+}$ embryos. Analysis of embryos at embryonic day 12 (E12) demonstrated that almost a quarter of them had intraabdominal hemorrhages with no clear macroscopic discernment of a liver (Fig. 4a). Genotyping verified that these embryos were $\mathrm{RelA}^{-1}$. Immunohistochemical analysis indicated very high immunoreactivity for cleaved-caspase 3 in the liver, signifying aberrant apoptosis (Fig. 4b). To further verify caspase- 3 activation and apoptosis, we probed for cleaved poly ADP-ribose polymerase (PARP)-1, a protein that is a target of activated caspase- 3 in its latent form. RelA ${ }^{-1-}$ embryonic liver had robust immunostaining of cleaved PARP-1 which was absent in RelA ${ }^{+/+}$livers. Isolated mouse embryonic fibroblasts (MEFs) were used to verify DNA recombination and RelA mRNA and protein depletion in RelA ${ }^{+/-}$and RelA ${ }^{-/}$cells (Fig. 4c-e). We failed to observe any shift in the RelA band $(65 \mathrm{kDa})$ using the Santa-Cruz antibody sc-372 that targets the TAD, suggesting that a truncated RelA is not produced in the genetically recombined cells. Furthermore, immunohistochemical analysis was performed using three different antibodies targeting the $\mathrm{N}$ - and $\mathrm{C}$-terminus domains of the RelA protein (Fig. 5). All three antibodies demonstrated robust RelA immunostaining in the liver, brain and vertebrae of $\mathrm{RelA}^{+/+}$embryos. Two antibodies targeting the Cterminus failed to show any staining in the $\mathrm{RelA}^{-1-}$ embryos whereas slight staining was observed in the liver with sc-109 antibody that recognizes the RHD at the N-terminus. The minor staining could be due to antibody cross-reactivity with other RHD-containing proteins such as RelB or C-Rel. To verify that the antibodies specifically recognized RelA, whole cell lysates from A549 cell line were immunoprecipitated with the three RelA antibodies and subjected to selected reaction monitoring- mass spectrometry analysis (SIDSRM-MS). Relative to IgG immunoprecipitates, there was 100-fold increase in RelA when immunoprecipitation was performed with sc-109 and ab7970 antibodies and approximately a 200 -fold increase in RelA when sc-372 antibody was used (Additional file 1: Figure S1). We interpret 


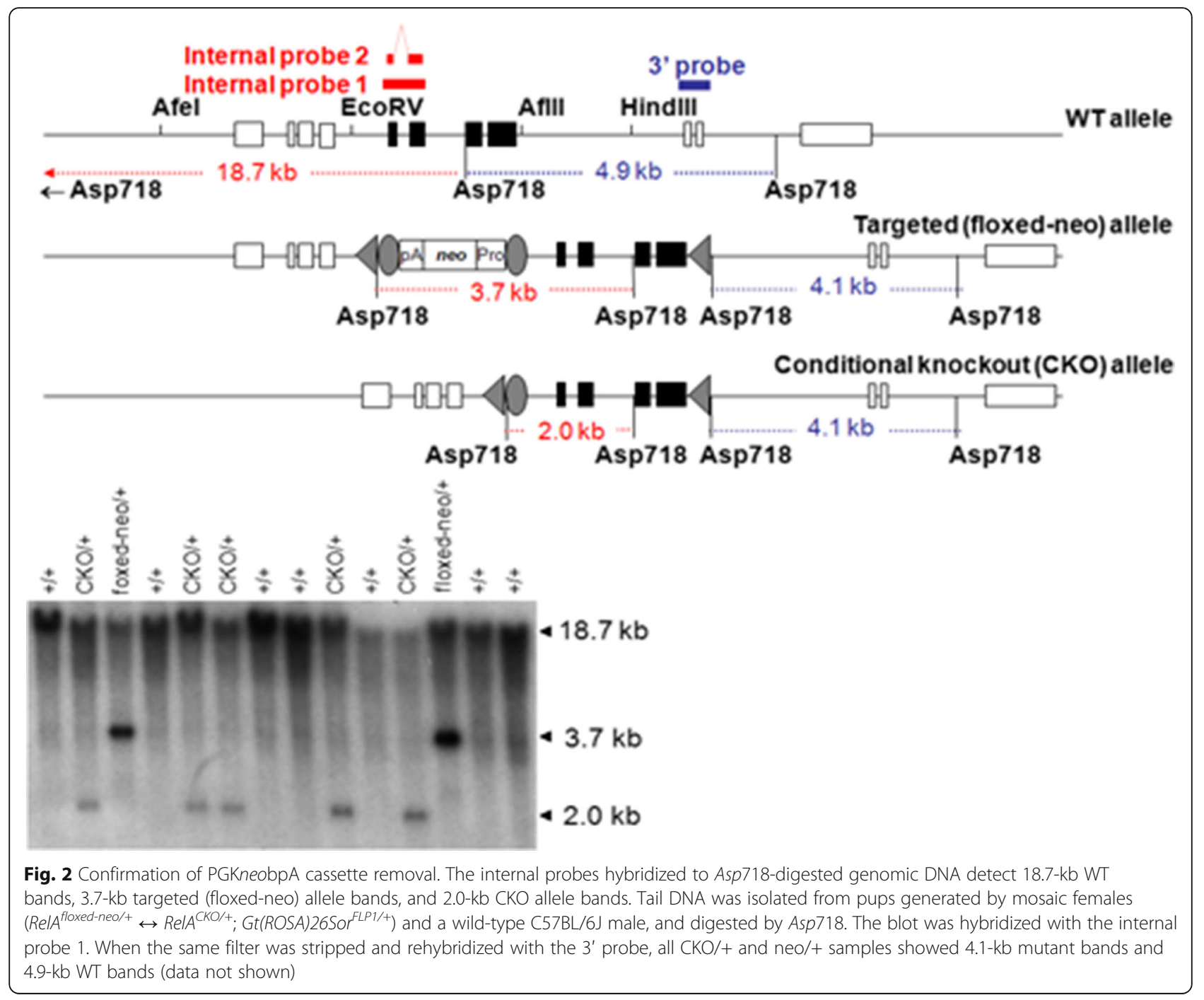

this to mean that all three antibodies recognize RelA and RelA ${ }^{\mathrm{CKO} / \mathrm{CKO}}$ cells that have undergone DNA recombination at the RelA alleles do not produce RelA. To determine whether Cre-mediated recombination can also occur in vitro, we transduced Cre via a lentivirus vector in RelA ${ }^{\mathrm{CKO} / \mathrm{CKO}}$ MEFs. In experiments infecting MEFs with only a multiplicity of infection (MOI) of 2, we observed a $90 \%$ reduction in RelA protein (Fig. 4f) with no shift in the RelA band. These data confirm that RelA can be depleted both in vitro using RelA $^{\mathrm{CKO} / \mathrm{CKO}}$ cells and in vivo using our RelA ${ }^{\mathrm{CKO} / \mathrm{CKO}}$ mice.

Our ultimate goal is to study the tissue-specific role of RelA in vivo. We have previously demonstrated that aortic fibroblasts produce significant amount of IL- 6 and MCP-1 that are NF-kB-dependent proteins, in vitro and the secretion of these cytokine/chemokine increases more than 2-fold in presence of monocytes [19, 20]. We have defined this interaction to be occurring in vivo in the aortic wall and contributing to aortic inflammation and to the development of aortic dissections [19]. To test the hypothesis that fibroblast-RelA contributes to aortic inflammation, we have developed fibroblastspecific RelA ${ }^{-1-}$ mice by crossing a tamoxifen inducible Col1 $\alpha 2$-promoter driven Cre mouse (Col1 $\alpha 2$-CreER) with RelA ${ }^{\mathrm{CKO} / \mathrm{CKO}}$ mice. Although the characterization of aortic fibroblast-RelA depletion and the role of fibroblastRelA in aortic inflammation are currently being investigated by our lab, we have described here the experiments validating RelA depletion that also occurs in skin fibroblast cells. After three generations of crosses, RelA ${ }^{\mathrm{CKO} / \mathrm{CKO}} \mathrm{Cre}+$ and $\mathrm{RelA}{ }^{\mathrm{CKO} / \mathrm{CKO}}$ Cre- littermates (Fig. 6a), which appeared normal and fertile, were treated with tamoxifen and their skin fibroblasts were isolated for characterization. Cre + fibroblasts had decreased levels of RelA in their cytoplasm (white arrows in Fig. 6b) and in response to TNF $\alpha$ stimulation, failed to accumulate RelA in the nucleus. We observed that only $50 \%$ of the fibroblast had decreased RelA immunostaining suggesting that 


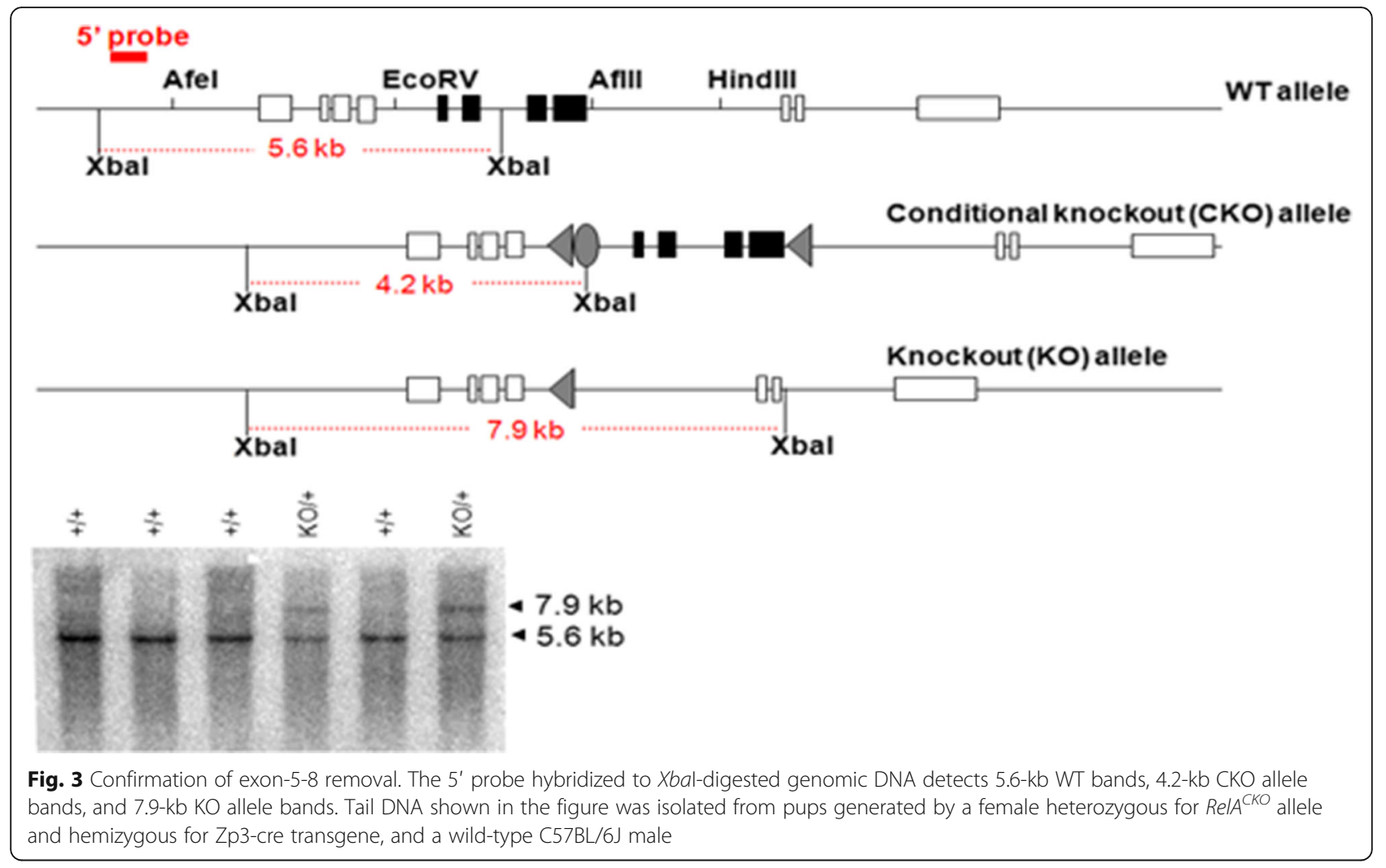

tamoxifen administration affected only half of the skin fibroblasts. Furthermore, RelA transcripts (Fig. 6c) and protein (Fig. 6d) were depleted by approximately $50 \%$ in $\mathrm{Cre}+$ fibroblasts. Collectively, these data demonstrated that Coll $\alpha 2-C r e E R$ is efficiently activated in half of the dermal fibroblasts by tamoxifen administration and can promote the recombination of RelA alleles in these cells leading to RelA depletion. Our future work will utilize this transgenic mouse to explore the role of fibroblast-RelA in the development of vascular inflammation and fibrosis in vivo.

\section{Conclusion}

In summary, we report the generation of a transgenic mouse containing RelA-flox alleles that recombine in the presence of active Cre recombinase to generate RelA $^{-1-}$ cells. This will be an important tool for studies investigating RelA signaling in adult tissues since it allows one to bypass a major limitation, death in utero, that accompanies whole-body $\operatorname{RelA}^{-1-}$. Furthermore, this will be a valuable mouse model for exploring the role of RelA in development, an under-explored area due to a lack of appropriate tools for investigation.

\section{Methods}

Construction of the RelA conditional knockout vector A 7.2-kb AfeI-HindIII DNA fragment (RelA 5' upstream-intron 8 region) was isolated from a bacterial artificial chromosome (BAC) clone, RP24-329M16 (obtained from the Children's Hospital Oakland Research Institute), and subcloned into pBluescript II SK (-) (Agilent, Santa Clara, CA) between SmaI and HindIII sites. A PGKneobpA cassette [21] flanked by an frt site on one end and an frt-loxP sequence on the other was inserted into the unique EcoRV site in intron 4 in reverse orientation relative to the orientation of RelA transcription via blunt-end ligation. Oligonucleotides for creating a DNA fragment containing a loxP site with an inserted Asp 718 site and two AflII ends were synthesized and annealed, and inserted into a unique $A f l \mathrm{II}$ site in intron 8. Finally, MC1tkpA cassette was added into the SalI site of the multiple cloning site region of the pBluescript for the enrichment of homologous recombinants via negative selection [22].

Genetic engineering of mouse embryonic stem (ES) cells The targeting vector was propagated, purified by cesium banding, and linearized by NotI. A total of $1 \times 10^{7}$ cells suspended in DPBS was electroporated with $25 \mu \mathrm{g}$ DNA using Gene Pulser $(500 \mu \mathrm{F} / 230 \mathrm{~V}$, BIO-RAD, Hercules, CA). The cells were then cultured in $200 \mu \mathrm{g} / \mathrm{ml}$ Geneticin (G418) and $200 \mathrm{nM}$ 1-(2-deoxy-2-fluoro-1-D-arabinofuranosyl)-5-iodouracil (FIAU) for 9 days. ES cell colonies that survived the G418-FIAU drug selection were expanded in three sets of 96-well plates. One set of the cells was 


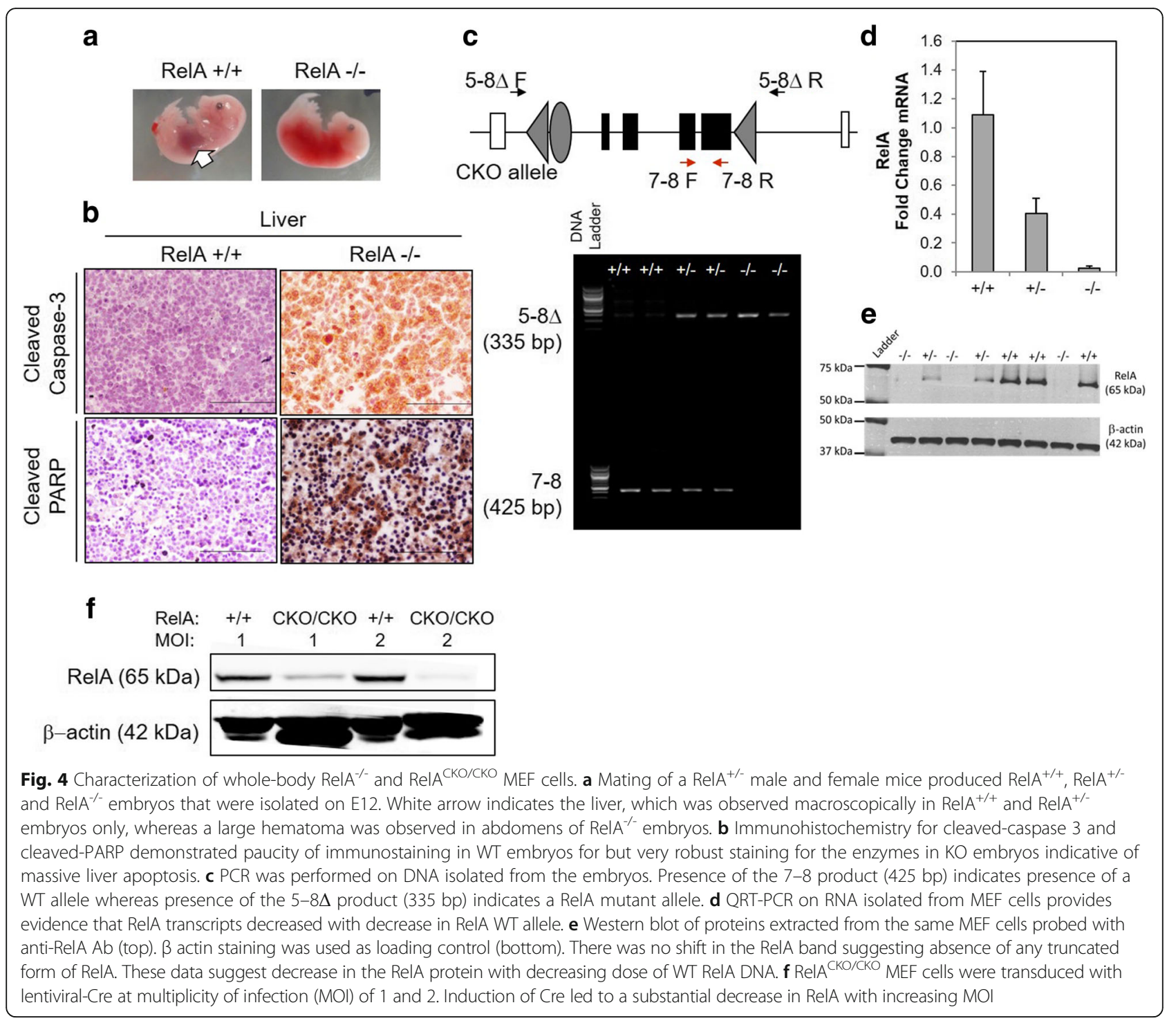

cryopreserved for blastocyst injection; the other two sets were processed for Southern blot analysis. The 5' and 3' flanking probes were hybridized to EcoRV-digested DNA and Asp718-digested DNA, respectively.

\section{Chimera production and animals}

Correctly targeted ES cell clones were injected into C57BL/6J blastocysts and injected embryos were transferred to 2.5-day-post-coitum pseudo-pregnant Swiss Webster female mice. All animal work was approved by UTMB's IACUC committee.

\section{Genotyping of the mouse using genomic Southern blot} Mice were genotyped by Southern blot analysis of tail DNA with 5'-flanking, 3'-flanking, and/or internal probes. All the probe fragments were generated by PCR, and subcloned into a T-vector. 5'-flanking probe: A 529-bp DNA was amplified form the BAC DNA with forward primers, TTGTGGGTAGCTGTGGTCAA, and reverse primer, CCAGCACTCCAGAAGAAAGG. 3'-flanking probe: A 468-bp DNA was amplified from the BAC DNA with forward primers, GGGAGAAGTGCAGCCCGGC, and reverse primer, CCCGGCCTCCCCCTGAGAA. Internal probe 1: A 561-bp DNA was amplified from the BAC DNA with forward primers, GATCCAGTGTGTGAAGA AGC, and reverse primer, GGTTATCAAAAATCGGAT GT. Internal probe 2: A 209-bp DNA was amplified from a pool of cDNA originating from mouse embryonic fibroblast RNA with forward primers, GATCCAGTGTGTG AAGAAGC, and reverse primer, GGTTATCAAAAATC GGATGT. Genomic DNA digested by appropriate restriction enzymes was separated on agarose/TAE gels, and blotted onto Hybond-XL membranes (GE Healthcare, Waukesha, WI). Blots were hybridized at $65{ }^{\circ} \mathrm{C}$ overnight 


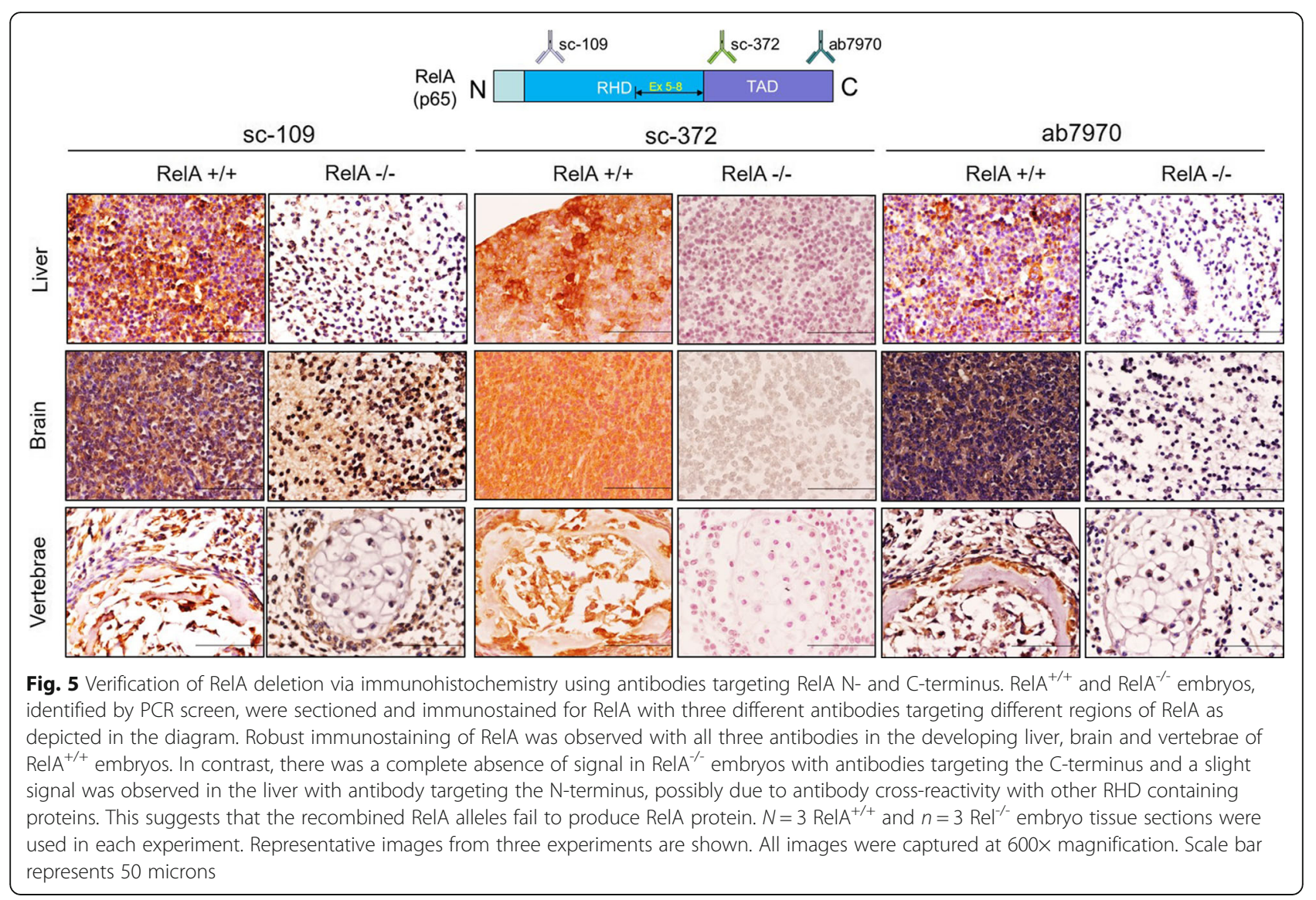

with radiolabeled and pre-associated probes in $1 \mathrm{M}$ sodium chloride, $1 \%$ SDS, $10 \%$ dextran sulfate with 100 $\mu \mathrm{g} / \mathrm{ml}$ salmon sperm DNA; and washed at $65{ }^{\circ} \mathrm{C}$ in $0.2 \mathrm{X}$ SSC, $0.1 \%$ SDS.

\section{Generation of MEF cells and genotyping of the mouse using PCR}

MEF cells were generated as described previously [23]. DNA was isolated from tail biopsies or MEF cells using phenol:chloroform extraction and ethanol precipitation followed by PCR. Briefly, tail biopsies or $500 \mathrm{~K}$ cells were incubated with $700 \mu \mathrm{l}$ of digestion buffer $(50 \mathrm{mM}$ Tris$\mathrm{HCl}, \mathrm{pH}$ 8; $100 \mathrm{mM}$ EDTA; $100 \mathrm{mM} \mathrm{NaCl} ; 1$ \% SDS; $350 \mu$ g Proteinase K) overnight at $55{ }^{\circ} \mathrm{C}$. Next morning, $700 \mu \mathrm{l}$ phenol:cholorform (Sigma) was added to each sample, samples were vortexed and centrifuged at 13,000 RPM for $10 \mathrm{~min}$. Approximately $500 \mu \mathrm{l}$ of supernatant was collected and $2 \times$ as much ethanol was added to precipitate the DNA. The mixture was centrifuged, the supernatant discarded and DNA pellet was re-suspended in $50 \mu \mathrm{l}$ of TE buffer. PCR reaction was set up using the following primers: 5-8 $\mathbf{\Delta} \mathbf{~ F}$, GCCGGCCAGGCAGCTC TTAC, and 5-8 $\boldsymbol{\Delta} \mathbf{R}$, GGCCAGTCACCATGGCCAGC, provide a 335 bp product only when RelA ${ }^{\mathrm{CKO}}$ allele undergoes recombination and 7-8 F, ACACTGCCGAG
CTCAAGATC, and 7-8 $\mathbf{R}$, AGCTGCATGGAGACTC GAAC, provide a 425 bp product when WT RelA is present. Presence of RelA ${ }^{\mathrm{CKO}}$ alleles was determined by RelAFlx F, TGCAAACAGACCTCCTTTGTCTTGA, and RelAFlx R, TCCTGAGACCAGACTCCTCCTCC, primers which provides a $450 \mathrm{bp}$ product if the CKO allele is present or $270 \mathrm{bp}$ product for a WT allele. PCR reaction was denatured at $94{ }^{\circ} \mathrm{C}$ for 2 min and subjected to 35 cycles of $30 \mathrm{~s}$ at $94{ }^{\circ} \mathrm{C}, 30 \mathrm{~s}$ at $54{ }^{\circ} \mathrm{C}$, and $60 \mathrm{~s}$ at $72{ }^{\circ} \mathrm{C}$. The PCR products were separated on a $1 \%$ agarose gel containing ethidium bromide and imaged on a UV transilliumintor. Colla2-CreER mice were genotyped according to instructions by The Jackson Laboratory (stock no. 016237).

\section{Quantitative RT-PCR (QRT-PCR)}

RNA was extracted from MEF cells using Trizol Reagent (Life Technologies, 15596-026) and was quantified using NanoDrop 2000 (Thermo Scientific). $1 \mu \mathrm{g}$ RNA was reverse transcribed to cDNA using SuperScript III FirstStrand Synthesis System in a $20 \mu$ reaction according to the manufacturer's instruction (Invitrogen, 18080-51). cDNA was diluted 1:2 and $3 \mu \mathrm{l}$ of the product was used in a $30 \mu \mathrm{l}$ reaction mixture containing $15 \mu \mathrm{l}$ SybrGreen mix and $500 \mathrm{nM}$ final concentration of RelA forward, 


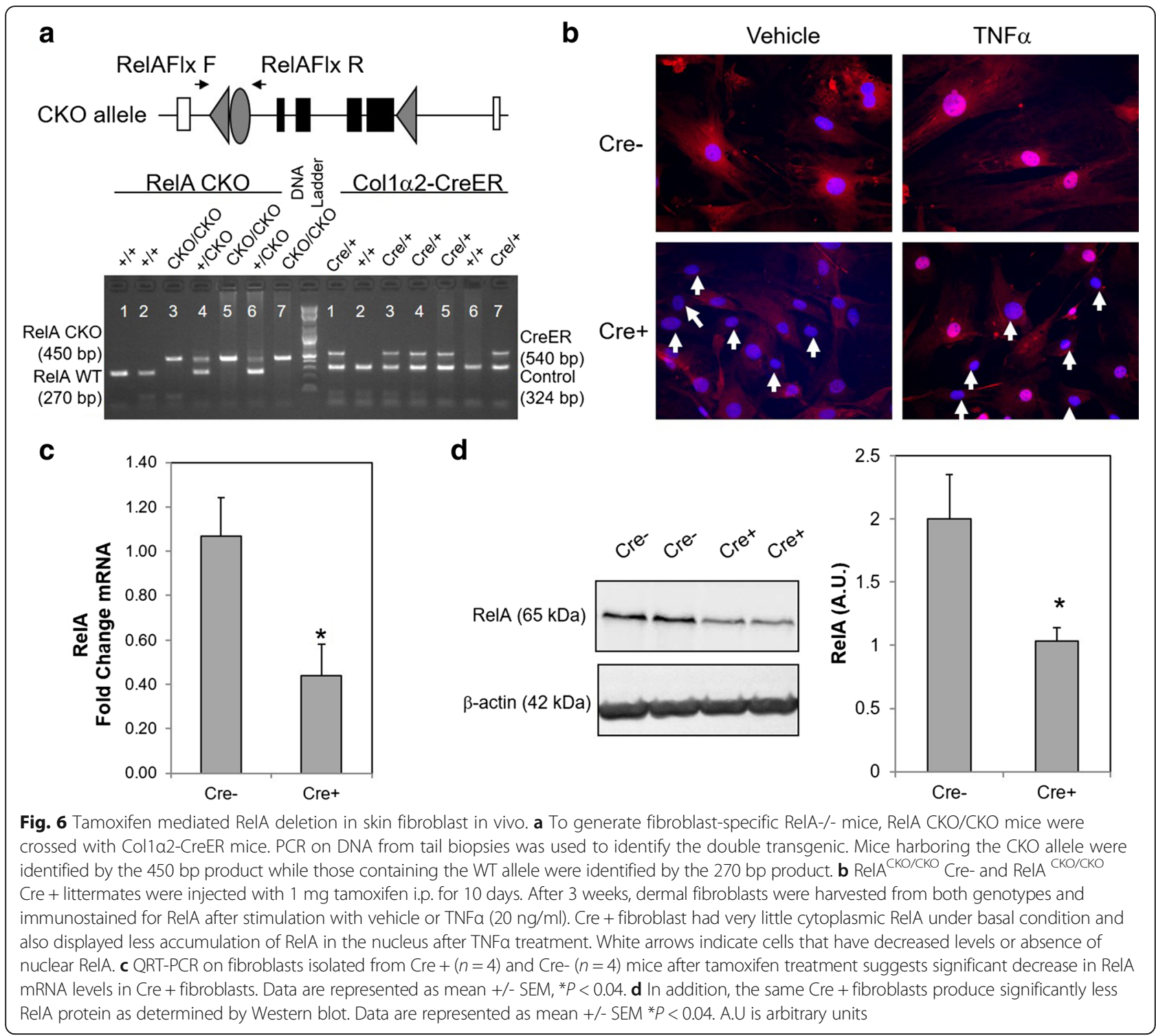

CCGGGATGGCTACTATGAGG, and RelA reverse, TCTTCACACACTGGATCCCC, primers or 18s rRNA forward, AGTCCCTGCCCTTTGTACACA, and 18s rRNA reverse, CGATCCGAGGGCCTCACTA, primers. The reaction mixtures were aliquoted into a Bio-Rad 96well PCR plate and sealed. The plates were denatured at $95{ }^{\circ} \mathrm{C}$ for $3 \mathrm{~min}$ followed by 40 cycles of $15 \mathrm{~s}$ at $95{ }^{\circ} \mathrm{C}$, $60 \mathrm{~s}$ at $60{ }^{\circ} \mathrm{C}$ and $1 \mathrm{~min}$ at $72{ }^{\circ} \mathrm{C}$. PCR products were subjected to melting curve analysis to ensure that a single product was produced. Change in gene expression was determined using $\Delta \Delta \mathrm{CT}$ method. RelA mRNA was normalized to $18 \mathrm{~s}$ rRNA.

\section{Immunohistochemistry}

Mouse embryos were formalin fixed and paraffin embedded. Tissue sections $(6 \mu \mathrm{m})$ were deparaffinized and rehydrated; antigen retrieval was performed with $10 \mathrm{mM}$
Sodium Citrate, pH6 before blocking with $5 \%$ goat serum and incubation with anti-RelA C-terminus (Santa Cruz sc-3702, 1:200, or Abcam ab7970, 1:300), anti-RelA N-terminus (Santa Cruz sc-109, 1:100), anti-cleaved capase 3 (Cell Signaling 5A1E, 1:100) or anti-cleaved PARP antibody (Cell Signaling D64E10, 1:50) overnight at $4{ }^{\circ} \mathrm{C}$. A biotinylated secondary antibody and avidin-biotin complex (Vector Labs, PK6101) was used to amplify the signal and DAB substrate (Vector Labs, SK4100) was used to detect the antigen-antibody complex. Images were obtained at $600 \times$ magnification using a Nikon digital camera DXM1200F attached to a Nikon Eclipse 80i microscope.

\section{Semiquantitative Western blot}

Whole cell protein lysates were extracted from MEF cells using RIPA buffer containing protease inhibitor 
cocktail. The resultant protein mixture was fractionated by $10 \%$ SDS-polyacrylamide gel electrophoresis and transferred to a PVDF membrane. After blocking with $5 \%$ milk in TBS-tween buffer, the membrane was incubated with rabbit anti-RelA antibody (Santa Cruz sc-372, 1:1000) and mouse anti-beta actin (Sigma A5316, 1:10,000) overnight at $4{ }^{\circ} \mathrm{C}$. Membranes were washed with TBS-tween before being incubated with IRDye 800-conjugated anti-rabbit and IRDye 700 conjugated anti-mouse secondary antibodies. RelA and beta-actin bands were detected using Licor Odyssey infrared scanner. Band densities were quantified using Image $\mathrm{J}(\mathrm{NIH})$ and represented in arbitrary units.

\section{Tamoxifen mediated genetic recombination and fibroblast culture} RelA ${ }^{\mathrm{CKO} / \mathrm{CKO}}$ female mice were crossed with Col1 $\alpha 2$ CreER male mice (gift from Dr. Arjun Deb, UNC Chapel Hill), both on $\mathrm{C} 57 \mathrm{Bl} / 6$ background, for three generations to generate RelA ${ }^{\mathrm{CKO} / \mathrm{CKO}} \mathrm{Cre}+$ and RelA ${ }^{\mathrm{CKO} / \mathrm{CKO}} \mathrm{Cre}-$ animals. Adult male Cre + and Cre- mice, 3-4 weeks old, were injected with Tamoxifen (Sigma T5648) $1 \mathrm{mg} /$ day intraperitoneally for 10 days. Tamoxifen was dissolved in $10 \%$ ethanol and $90 \%$ corn oil for $10 \mathrm{mg} / \mathrm{ml}$ working solution. After another 3 weeks, mice were euthanized and tissues were harvested for characterization. Skin samples were incubated with $0.25 \%$ trypsin overnight at $4{ }^{\circ} \mathrm{C}$. The skin was then minced and digested in 0.14 Wunsch units/ml Liberase Blendzyme 3 (Roche) containing $1 \times$ antibiotic/antimycotic (Invitrogen) in DMEM/F12 media for $1-2 \mathrm{~h}$ at $37^{\circ} \mathrm{C}$. The skin was further dissociated and resuspended in DMEM/12 media containing 10 \% FBS, $1 \times$ antibiotic/antimycotic and nonessential amino acids (complete media). Cells were centrifuged at $1000 \times \mathrm{g}$ for 10 mins and the pellet was resuspended in complete media for culturing. After 3 passages, only surviving cells were dermal fibroblasts which were characterized via immunofluorescence, QRT-PCR and Western blot.

\section{Statistical analysis}

Student's t-test (2-tail, assuming unequal variance) was use to analyze difference between two groups. $P<0.05$ was considered statistically significant.

\section{Additional file}

Additional file 1: Figure S1. Whole cell lysates of A549 cells were prepared by hypotonic lysis in $50 \mathrm{mM} \mathrm{NaCl}, 10 \mathrm{mM}$ Tris, pH 7.8, 2 mM EDTA, 1 \% IGEPALCA-630, with protease inhibitor cocktail. Equal volumes of whole cell lysates were immunoprecipitated overnight with $4 \mu \mathrm{g}$ of indicated antibody. Immunoprecipitates were captured on protein A magnetic beads and washed $4 \times$ in PBS. Samples were subjected to on-bead digestion and assayed for RelA using SID-SRM-MS [24]. Shown is mean +/- SD of RelA 756 signal relative to internal stable isotope standard (SIS). Note significant enrichment of RelA signal with each antibody. (PSD $150 \mathrm{~kb}$ )

\section{Abbreviations}

CKO: Conditional knock-out; NF-kB: Nuclear factor-kappa b; RHD: Rel homology domain; TAD: Transcription activation domain

\section{Acknowledgements}

We would like to thank Dr. Tom Wood, Dr. Iryna Pinchuk and Dr. Jun Yang for helpful discussions. Core laboratory support was provided by the Transgenic Mouse Facility and Recombinant DNA Laboratories of UTMB.

\section{Funding}

This work was supported by F30HL128036 (T.I.), PO1 Al068865 (A.R.B.) UTMB CTSA UL1TR001439 (A.R.B.), NIEHS P30 ES006676 (A.R.B.), and AHA 13GRNT17120070 (R.G.T.).

Availability of data and materials

The data on which this manuscript draws its conclusion is provided in the body of the manuscript.

\section{Authors' contributions}

$\mathrm{TI}, \mathrm{MW}$, and ARIII helped create the targeting vector and the conditional knockout allele mouse. TI and HS maintained the animal colonies and helped characterized the global knock out and fibroblast-specific knock out mouse. ARB and RGT conceived of the study, contributed to the experimental design, data interpretation and coordinated the project. All listed authors were involved in preparing the manuscript. All authors read and approved the final manuscript.

\section{Competing interests}

The authors declare that they have no competing interests.

\section{Consent for publication}

Not applicable.

\section{Ethics approval and consent to participate}

The University of Texas Medical Branch's Institutional Animal Care and Use Committee approved the animal work that is reported in this manuscript (protocol\# 0105020B)

\section{Author details}

${ }^{1}$ Departments of Biochemistry and Molecular Biology, University of Texas Medical Branch, Galveston, TX, USA. ${ }^{2}$ Institute for Translational Sciences, University of Texas Medical Branch, Galveston, TX, USA. Internal Medicine-Division of Endocrinology, University of Texas Medical Branch Galveston, TX, USA. ${ }^{4}$ Sealy Center for Molecular Medicine, University of Texas Medical Branch, MRB 8.128, 301 University Blvd, Galveston, TX 77555-1060, USA.

Received: 19 May 2016 Accepted: 13 September 2016

Published online: 23 September 2016

References

1. Baker RG, Hayden MS, Ghosh S. NF-kB, inflammation, and metabolic disease. Cell Metab. 2011:13:11-22.

2. Gilmore TD. Introduction to NF-kappaB: players, pathways, perspectives. Oncogene. 2006;25:6680-4

3. Brasier AR. The nuclear factor-kappaB-interleukin-6 signalling pathway mediating vascular inflammation. Cardiovasc Res. 2010:86:211-8.

4. Beg AA, Sha WC, Bronson RT, Ghosh S, Baltimore D. Embryonic lethality and liver degeneration in mice lacking the RelA component of NF-kappa B. Nature. 1995;376:167-70

5. Doi TS, Takahashi T, Taguchi O, Azuma T, Obata Y. NF-kappa B RelAdeficient lymphocytes: normal development of T cells and B cells, impaired production of $\lg A$ and $\lg G 1$ and reduced proliferative responses. J Exp Med. 1997:185:953-61.

6. Doi TS, Marino MW, Takahashi T, Yoshida T, Sakakura T, Old L, Obata Y. Absence of tumor necrosis factor rescues RelA-deficient mice from embryonic lethality. Proc Natl Acad Sci U S A. 1999;96:2994-9.

7. Van Antwerp DJ, Martin SJ, Kafri T, Green DR, Verma IM. Suppression of TNF-alpha-induced apoptosis by NF-kappaB. Science. 1996;274:787-9. 
8. Wang CY, Mayo MW, Korneluk RG, Goeddel DV, Baldwin AS. NF-kappaB antiapoptosis: induction of TRAF1 and TRAF2 and c-IAP1 and c-IAP2 to suppress caspase-8 activation. Science. 1998;281:1680-3.

9. Algül H, Treiber M, Lesina M, Nakhai H, Saur D, Geisler F, Pfeifer A, Paxian S, Schmid RM. Pancreas-specific RelA/p65 truncation increases susceptibility of acini to inflammation-associated cell death following cerulein pancreatitis. J Clin Invest. 2007;117:1490-501.

10. Heise N, De Silva NS, Silva K, Carette A, Simonetti G, Pasparakis M, Klein U. Germinal center B cell maintenance and differentiation are controlled by distinct NF-kB transcription factor subunits. J Exp Med. 2014;211:2103-18.

11. Weingarten-Gabbay S, Elias-Kirma S, Nir R, Gritsenko AA, Stern-Ginossar N, Yakhini Z, Weinberger A, Segal E. Comparative genetics. Systematic discovery of cap-independent translation sequences in human and viral genomes. Science. 2016;351:240.

12. Narayanan R, Klement JF, Ruben SM, Higgins KA, Rosen CA. Identification of a naturally occurring transforming variant of the p65 subunit of NF-kappa B. Science. 1992;256:367-70.

13. Lyle R, Valleley EM, Sharpe PT, Hewitt JE. An alternatively spliced transcript, p65 delta 2, of the gene encoding the p65 subunit of the transcription factor NF-kappa B. Gene. 1994;138:265-6,

14. Epelman S, Lavine K, Beaudin AE, Sojka DK, Carrero JA, Calderon B, Brija T, Gautier EL, Ivanov S, Satpathy AT, et al. Embryonic and adult-derived resident cardiac macrophages are maintained through distinct mechanisms at steady state and during inflammation. Immunity. 2014;40:91-104.

15. George SH, Gertsenstein M, Vintersten K, Korets-Smith E, Murphy J, Stevens ME, Haigh JJ, Nagy A. Developmental and adult phenotyping directly from mutant embryonic stem cells. Proc Natl Acad Sci U S A. 2007;104:4455-60.

16. Farley FW, Soriano P, Steffen LS, Dymecki SM. Widespread recombinase expression using FLPeR (flipper) mice. Genesis. 2000;28:106-10.

17. de Vries WN, Binns LT, Fancher KS, Dean J, Moore R, Kemler R, Knowles BB. Expression of Cre recombinase in mouse oocytes: a means to study maternal effect genes. Genesis. 2000;26:110-2.

18. Lewandoski M, Wassarman KM, Martin GR. Zp3-cre, a transgenic mouse line for the activation or inactivation of loxP-flanked target genes specifically in the female germ line. Curr Biol. 1997;7:148-51.

19. Tieu BC, Ju X, Lee C, Sun H, Lejeune W, Recinos A, Brasier AR, Tilton RG. Aortic adventitial fibroblasts participate in angiotensin-induced vascular wall inflammation and remodeling. J Vasc Res. 2011;48:261-72.

20. Tieu BC, Lee C, Sun H, Lejeune W, Recinos A, Ju X, Spratt H, Guo DC, Milewicz D, Tilton RG, Brasier AR. An adventitial IL-6/MCP1 amplification loop accelerates macrophage-mediated vascular inflammation leading to aortic dissection in mice. J Clin Invest. 2009:119:3637-51.

21. Soriano P, Montgomery C, Geske R, Bradley A. Targeted disruption of the c-src proto-oncogene leads to osteopetrosis in mice. Cell. 1991;64:693-702.

22. Mansour SL, Thomas KR, Capecchi MR. Disruption of the proto-oncogene int-2 in mouse embryo-derived stem cells: a general strategy for targeting mutations to non-selectable genes. Nature. 1988;336:348-52.

23. Xu J. Preparation, culture, and immortalization of mouse embryonic fibroblasts. Curr Protoc Mol Biol. 2005; Chapter 28:Unit 28.21.

24. Zhao Y, Widen SG, Jamaluddin M, Tian B, Wood TG, Brasier AR. Quantification of Activated NF-kB/RelA Complexes Using ssDNA Aptamer Affinity-Stable Isotope Dilution-Selective Reaction Monitoring-MS. Mol Cellular Proteomics. 2011;10(6):1-16.

\section{Submit your next manuscript to BioMed Central and we will help you at every step:}

- We accept pre-submission inquiries

- Our selector tool helps you to find the most relevant journal

- We provide round the clock customer support

- Convenient online submission

- Thorough peer review

- Inclusion in PubMed and all major indexing services

- Maximum visibility for your research

Submit your manuscript at www.biomedcentral.com/submit 